\title{
Energy Optimal Trajectories in Human Arm Motion Aiming for Assistive Robots
}

\author{
Lelai Zhou ${ }^{1}$ Shaoping Bai ${ }^{2}$ Yibin $\mathrm{Li}^{1}$ \\ ${ }^{1}$ School of Control Science and Engineering, Shandong University, Jinan, 250061, China. E-mail: \{zhoulelai, \\ liyb\}@sdu.edu.cn \\ ${ }^{2}$ Department of Mechanical and Manufacturing Engineering, Aalborg University, Aalborg, Denmark. E-mail: \\ shb@m-tech.aau.dk
}

\begin{abstract}
The energy expenditure in human arm has been of great interests for seeking optimal human arm trajectories. This paper presents a new way for calculating metabolic energy consumption of human arm motions. The purpose is to reveal the relationship between the energy consumption and the trajectory of arm motion, and further, the acceleration and arm orientation contributions. Human arm motion in horizontal plane is investigated by virtue of Qualisys motion capture system. The motion data is post-processed by a biomechanical model to obtain the metabolic expenditure. Results on the arm motion kinematics, dynamics and metabolic energy consumption, are included.
\end{abstract}

Keywords: metabolic cost; human arm motion; energy consumption

\section{Introduction}

Anthropomorphic robotic arms can be used for service tasks. While the motion of the robotic arm can be controlled with different methods, trajectories that enable human-like energy efficient arm movement are desirable. The principles of human arm motion, to this end, can be adopted in the control of anthropomorphic robotic arms. Upon basic definition, a human arm has seven dof (degrees-of-freedom), with three in the shoulder, two in the elbow, and two in the wrist. The human arm has one redundant dof, which enables its infinite possible trajectories for an intending motion. For instance, when a person needs to pick up an apple, the arm unintentionally chooses one trajectory from a huge amount. The arm can have different orientations with the hand fixed at one point. However, for any given pair of end points, the trajectories that a human chooses are highly predictable (Flash and Hogan, 1985), (Atkeson and Hollerbach, 1985), even though we choose the particular trajectories unintentionally.
Over years, researchers have studied the mechanism of unintentional chosen trajectories of human arm. The kinematic analysis cannot perfectly explain the planning mechanism of human arms, because the activation of the individual muscles generating forces cannot be specified. One effective approach to investigating the planning mechanism is to examine the mechanical and physiological properties of a muscle, and to investigate the behavior of individual muscles in human arm trajectories (Kashima et al., 2002), (Fagg et al., 2002), (Georgopoulos et al., 1986). Different optimization criteria based on the minimum energy were proposed (Alexander, 1997), (Buchanan and Shreeve, 1996), (Lan, 1997). A minimum energy cost hypothesis for human arm trajectories was presented and tested (Alexander, 1997). An approach for predicting metabolic cost in the planar human arm motion was proposed to predict energy optimal trajectories (Zhou et al., 2011).

Past observations on multi-joint human arm trajectories obtained from restricted horizontal planar move- 
ments measured with apparatus have shown that human point to point arm motion trajectories are essentially straight with bell-shaped velocity profiles (Abend et al., 1982), (Morasso, 1981). Many scholars have made great efforts to explain observed trajectories as solutions to optimization problems. Optimization criteria have been proposed including minimum jerk theory (Flash and Hogan, 1985), minimum travel cost theory (Rosenbaum et al., 1995), minimum isometric torque derivative (Kashima and Isurugi, 1998), and averaged specific power (Secco et al., 2005).

We have investigated unrestrained human arm trajectories between point targets using Qualisys motion capture system. Our studies focus on the energy consumption in real human arm motions. Subjects were instructed to perform planar movements between several pairs of points. In this paper, a method to capture and process human arm motion data is presented, through which, kinematic and dynamic properties are obtained. By virtue of Anybody software, energy consumption during arm motion is deduced. Upon above results, the energy behavior of human arm motion is analyzed, the relationship between metabolic energy consumption and trajectory, hand acceleration as well as arm orientation is deduced.

\section{Methodology}

As shown in Fig. 1, the system for the experiments consists of Qualisys Motion Capture System, Qualisys Track Manager, and AnyBody Modeling System software. Qualisys Motion Capture System consists of eight cameras surrounding the subject to reconstruct the motion in the computer. Qualisys Track Manager is a post processing software to build model on the basis of capture data. AnyBody Modeling System is a kind of biomechanical analysis system.

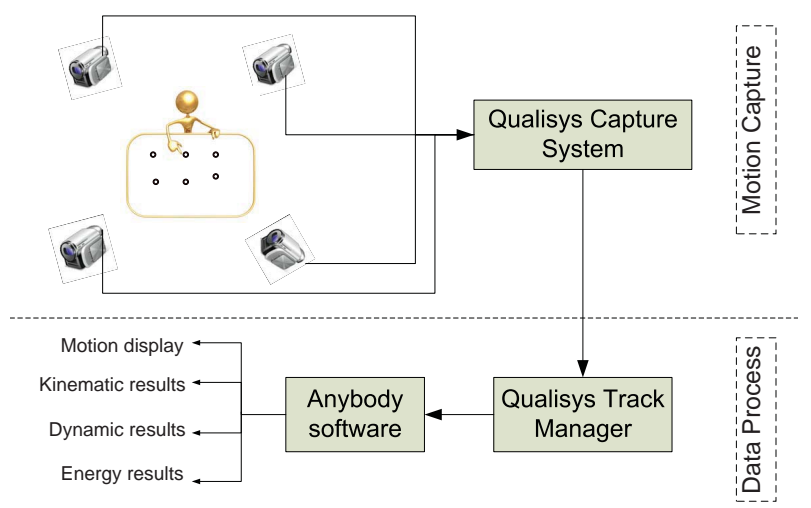

Figure 1: Experimental system for human arm motion capture and analysis

\subsection{Experimental Procedure}

A human subject is seated in front of an experimental table, the height of which is adjusted to just below his shoulder joints. Six points are predefined on a planar board, as indicated in Fig. 2. The planar board is the same as used by Abend et al. (Abend et al., 1982). The coordinate system of this workspace is shown in Fig. 2. $\mathrm{X}$-axis, which is perpendicular to the line connecting the subject's both shoulders, is located on his shoulder. The origin is defined at point $S_{1}$ on the board.

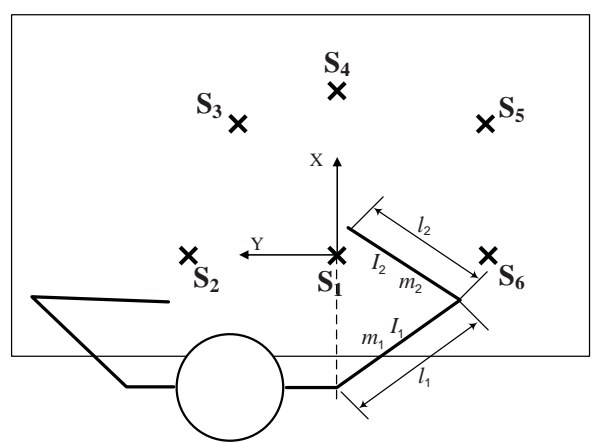

Figure 2: Experimental board with coordinate system and 6 points performed as targets to be reached by human subjects with forefingertip

Subjects move the forefinger with marker from one point to another at normal speed, while keeping the arm horizontal at the subjects' shoulder level, such that the movements are confined to elbow and shoulder rotations in a horizontal plane. Three types of paths are to be followed by human subjects, the straight, curved, and $S$-shaped paths. No specific instructions are given with regard to accuracy of the movement, except the general shape of the path. Arm movement for each path is repeated 10 times to get sufficient data for further analysis.

\subsection{Marker Arrangement}

The choice of marker location has shown great variability, especially on the upper arm. The marker arrangement depends on both accuracy and visibility. The main concern is to avoid excessive errors due to skin movement under the markers, and to keep the markers in view at all times (Anglin and Wyss, 2000), (Kontaxis et al., 2009). As indicated in Fig. 2, one marker is set on the forefinger-tip to be recognized as an end point of hand, the hand is identified by one marker on the forefinger basic styloid process, the wrist is identified by one marker on each styloid process, the elbow is marked both at the lateral and medial epicondyle, the 
trunk is identified by two markers on each shoulder, two markers on up body, and one on back neck.

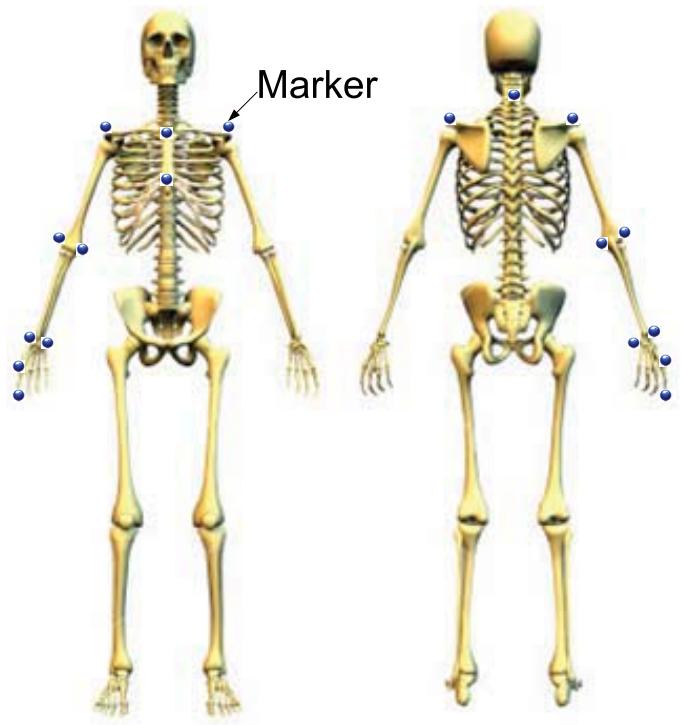

Figure 3: Marker arrangement on a human subject. Totally, 11 markers are used, with 7 markers on the right arm, 3 markers on the trunk, and 1 marker on the left shoulder, opposite to its counterpart at the right shoulder

\subsection{Motion Capture System}

The arm motion is recorded by Qualisys Motion Capture system. Retro-reflective markers are placed on the subject. The system tracks the markers' positions, while arm is moving. Marker data is displayed in realtime on the monitor. By connecting a video camera, it is possible to present synchronized video image along with the acquired data. QTM, Qualisys proprietary tracking software, is designed to work seamlessly with any model of Qualisys camera, ensuring fast and precise data collection. The system enables users to perform capture of data in real-time, with minimal latency (QTM, 2008).

\subsection{Post-processing System}

The AnyBody Modeling system is used as the postprocessing system. It is a software system for analyzing the mechanics of the live human body working in consistent with its environment. The environment is defined in terms of external forces and boundary conditions, and the user may impose any kind of posture or motion for the human body - either from scratch or from a set recorded motion data. AnyBody calculates the mechanical properties for the body-environment system. With the markers data from Qualisys, an arm model is constructed in AnyBody modeling system, through which the kinematic and dynamic properties of human arm motion can be achieved.

\subsection{Human Arm Parameters}

A human arm (Fig. 2) with two moveable joints is modeled. Parameters are measured or estimated from data taken from a published book (Winter, 1990) and given in Table 1.

\subsection{Paths for arm motion}

Total five cases refer to different pairs of points are shown in Fig. 4. In Case $A$, three paths including a straight path, a curved path, and a $S$-shaped path are defined, as direction indicated by the arrows from point $S_{1}$ to point $S_{4}$. In other cases, two paths of finger route are shown respectively in Fig. 4(b)- 4(e), indicate a straight and a curved path separately, as directions indicated by the arrows.

\section{Results}

The captured human arm trajectories are calculated and compared in this section.

\subsection{Dependence of Energy Consumption on Trajectory}

A typical example for kinematic and energy properties of three paths in Case $A$ is shown in Fig. 5. The line style of each profile is in accordance with that of the trajectory in Fig. 4(a). Since the arm movements are confined in horizontal plane, the potential energy of the arm remains unchanged. The movements require only work to accelerate and decelerate arm segments. The calculations of energy consumption during the movements are significantly simplified in terms of neglecting potential energy change.

In order to avoid calculating muscle metabolism by dealing with complex formulas as in (Woledge et al., 1985), (Winters and Stark, 1985), (Ma and Zahalak, 1991), the arm kinetic energy is calculated by means of AnyBody Modeling System, as shown in Fig. 5(f). Based on the kinetic energy profile, muscle metabolism can be calculated by means of method depicted in Fig. 6 .

The variation of an arm kinetic energy with respect to time is shown in Fig. 6. From time $t=0$ to $t=t_{1}$, the muscles in the arm do positive work as the arm kinetic energy is increasing, therefore the muscle metabolism in this period is $K_{1}$. From time $t=t_{1}$ 
Table 1: Parameters of a human arm.

\begin{tabular}{lcccc}
\hline Description & Parameters & Value & Unit \\
\hline Mass of upper arm & $m_{1}$ & 2.0 & $\mathrm{~kg}$ & $*$ \\
Mass of lower arm & $m_{2}$ & 1.5 & $\mathrm{~kg}$ & \\
Moment of inertia & $I_{1}$ & 0.016 & $\mathrm{~kg} \cdot \mathrm{m}^{2}$ & \\
Moment of inertia & $I_{2}$ & 0.011 & $\mathrm{~kg} \cdot \mathrm{m}^{2}$ & \\
\hline Length of upper arm & $l_{1}$ & 0.30 & $\mathrm{~m}$ & $\star$ \\
Length of lower arm & $l_{2}$ & 0.32 & $\mathrm{~m}$ & \\
\hline Distance from center of mass to shoulder joint center & $l_{c 1}$ & 0.15 & $\mathrm{~m}$ & $\diamond$ \\
Distance from center of mass to elbow joint center & $l_{c 2}$ & 0.12 & $\mathrm{~m}$ & \\
\hline
\end{tabular}

* Data from (Winter, 1990)

* Measured data

$\diamond$ Estimated data from (Winter, 1990)

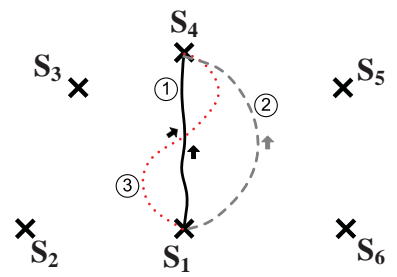

(a) Case $A$

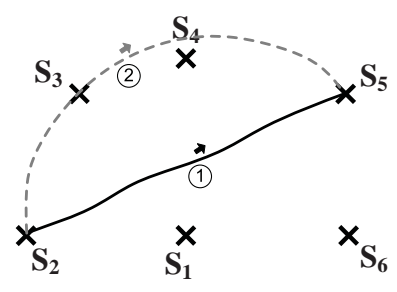

(b) Case $B$

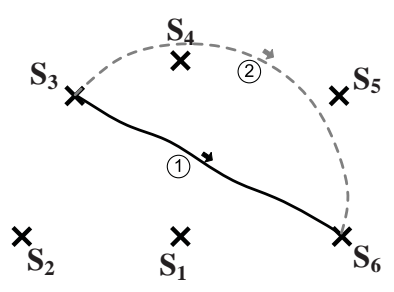

(c) Case $C$

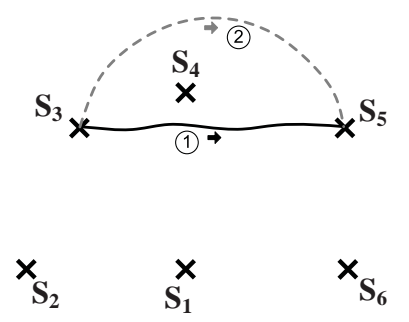

(d) Case $D$

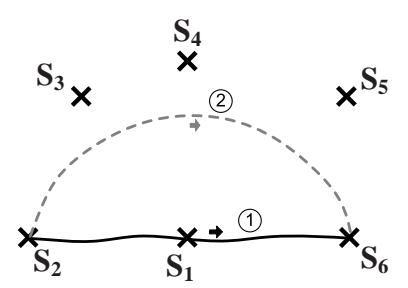

(e) Case $E$

Figure 4: Five cases of human arm motion

to $t=t_{2}$, the arm kinetic energy is decreasing, which requires the muscles to do negative work. The muscle metabolism in this period is $K_{2}$. And so forth, the muscle metabolism in periods $t=t_{2}$ to $t=t_{3}$ and $t=t_{3}$ to $t=t_{4}$ is $K_{3}$ and $K_{4}$ respectively. The total muscle metabolism during an arm movement is given by

$$
E=K_{1}+K_{2}+K_{3}+K_{4}
$$

where $E$ is total muscle metabolism as the energy consumption. by

In general, the muscle metabolism can be calculated

$$
E=\sum_{i=1}^{2 n} K_{i}=\sum_{i=1}^{2 n}\left|J_{i}-J_{i-1}\right|
$$

where $J_{i}$ and $J_{i-1}$ are the peak values of a kinetic energy curve, $n$ denotes the amount of kinetic energy peaks.

Table 2 shows the calculated energy consumption in terms of muscle metabolism along those paths as shown in Fig. 4. The minimal metabolic energy cost occurs in the straight trajectory in most of the cases except Case $C$. It is general knowledge that the shorter distance between two points requires less energy consumption than longer one, as proved by the data in Table 2. However in Case $C$, it is violated as the shorter trajectory required a bit more energy, there must be some other aspects that influence the energy consumption that we will discuss later.

As shown in Fig. 4, the distance between point $S_{3}$ and $S_{6}$ is less than the distance between point $S_{2}$ and $S_{5}$, and more or less the same as the distance between point $S_{2}$ and $S_{6}$, the metabolic energy costs for each straight trajectory are $2.33 \mathrm{~J}, 0.64 \mathrm{~J}$, and $1.78 \mathrm{~J}$ 

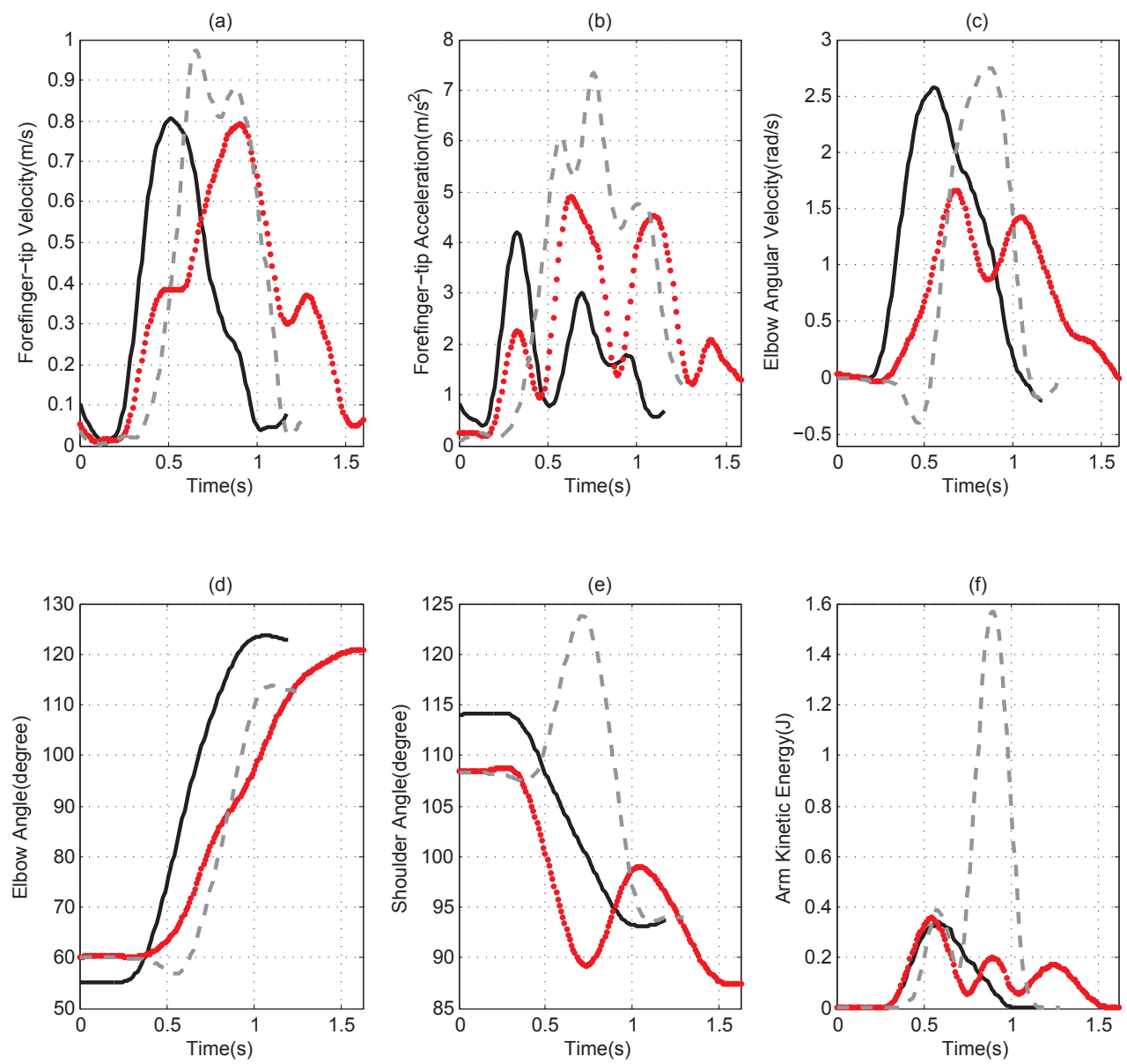

Figure 5: Kinematic and energy variations of three trajectories in Case $A$ : (a) forefinger-tip velocity, (b) forefinger-tip acceleration, (c) elbow angular velocity, (d) elbow joint angle, (e) shoulder joint angle, (f) arm kinetic energy.

respectively. It is obvious that the distance between two points cannot determine the metabolic energy consumption as the shorter distance requires more energy between $S_{3}$ and $S_{6}$. The results show strong dependence of energy consumption on arm trajectory.

\subsection{The Acceleration Contribution}

Three trajectories for arm movements generated by subjects are shown in Fig. 5. The metabolic energy consumptions for the straight, curved, and $S$-shaped trajectory in Case $A$ are $0.73 J, 3.74 J$, and $1.39 J$ respectively. In Case $A$, the curved trajectory requires much more energy than the other two. It is the most energy consumed trajectory among all the paths as shown in Table 2. Through comparing the forefingertip velocity profile (Fig. 5(a)), the forefinger-tip acceleration profile (Fig. 5(b)) and the arm kinetic energy profile (Fig. 5(f)), the acceleration profile of the curved trajectory(in gray color) has a much higher peak than the other two, and its velocity profile is also a bit higher. As the figures indicate, acceleration has a great contribution to arm kinetic energy, which conducts to metabolic energy cost subsequently.

Arm movements of two trajectories for Case $B$ are compared in Fig. 7. The curved trajectory (dash line) has a higher peak than the straight one (Fig. 7(b)), leads to higher kinetic energy (Fig. 7(f)), hence requires more energy consumed in the muscles (which are 1.33J and $0.64 J$ separately as in Table 2).

The peaks of forefinger-tip acceleration for curved trajectory (dash line) along Case $A$ and Case $B$ are $7.4 \mathrm{~m} / \mathrm{s}^{2}$ (Fig. 5(b)) and $3.9 \mathrm{~m} / \mathrm{s}^{2}$ (Fig. 7(b)) respectively. Note that their metabolic energy costs are $3.74 \mathrm{~J}$ and $1.33 \mathrm{~J}$, it is distinctly that acceleration has a positive correlation with the metabolic energy consumption. 
(a)

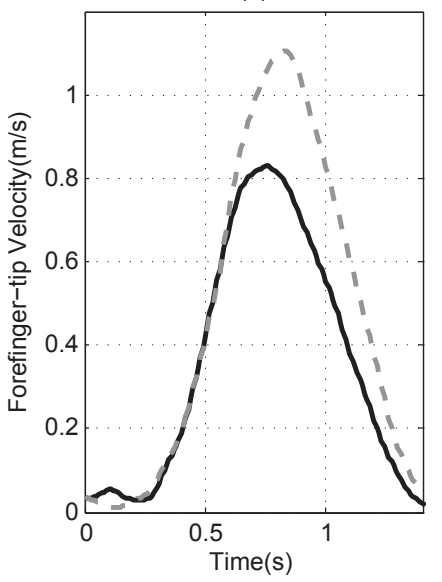

(d)

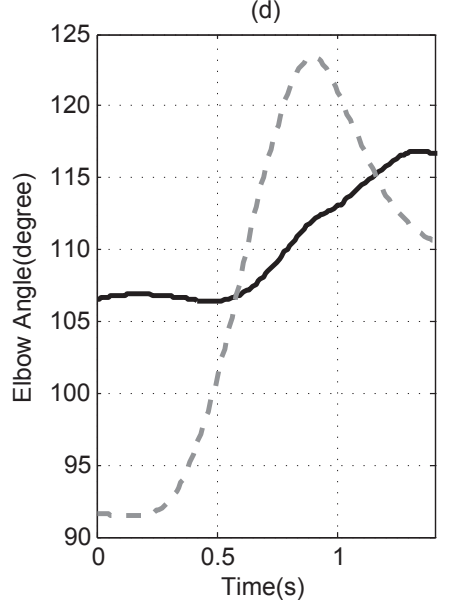

(b)

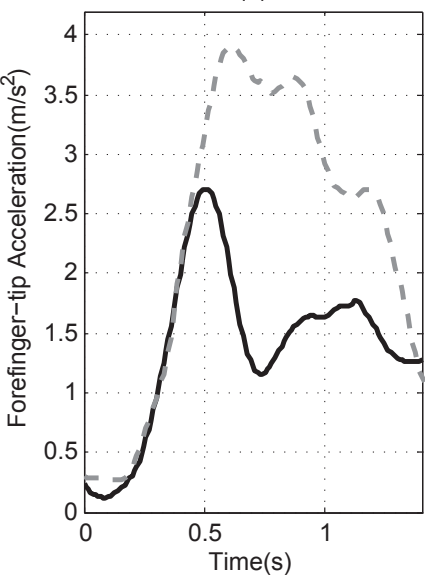

(e)

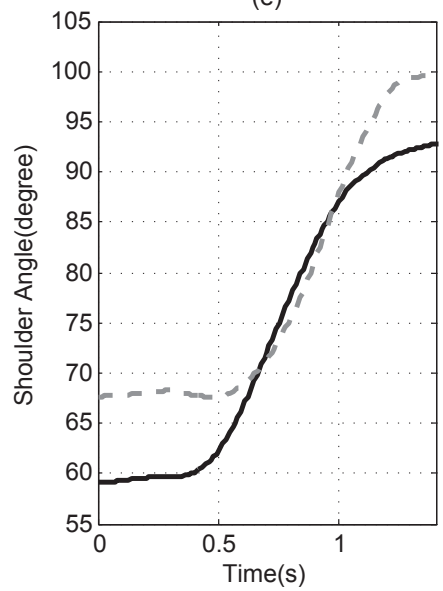

(c)

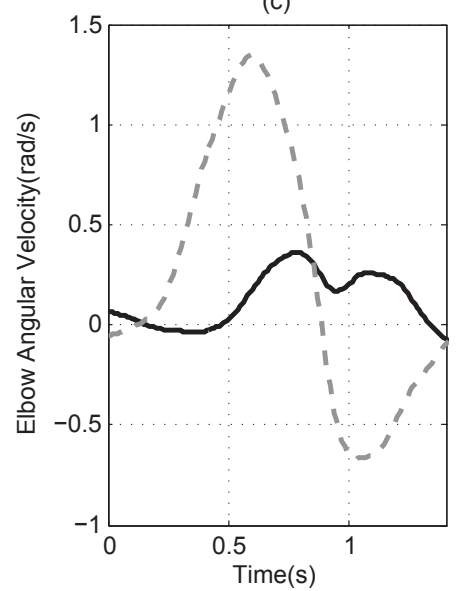

(f)

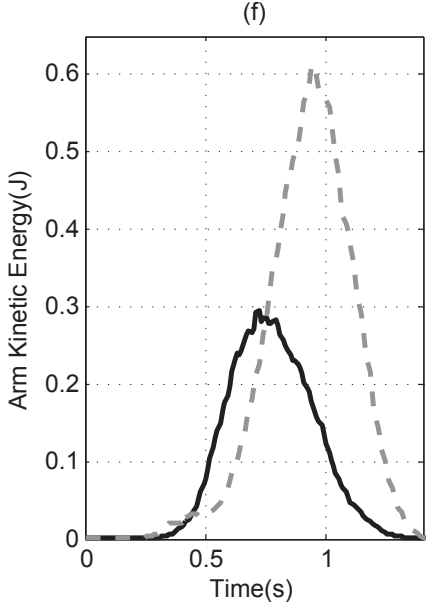

Figure 7: Measured kinematic and energy property profiles of two trajectories in Case $A$ : (a) forefinger-tip velocity, (b) forefinger-tip acceleration, (c) elbow angular velocity, (d) elbow joint angle, (e) shoulder joint angle, (f) arm kinetic energy profiles.

\subsection{Arm Orientation}

Comparisons of elbow and shoulder joint angles and kinetic energy for movements along two trajectories in Case $C$, Case $D$, and Case $E$ are shown in Fig. 8 to Fig. 10. As depicted in Fig. 4, Case $B$ and Case $E$ are started from the same point $S_{2}$ to different end points, $S_{5}$ and $S_{6}$ separately. The elbow and shoulder angle for each path (Fig. 7(d),(e) and Fig. 10(a),(b)) show different arm orientations of subjects during the movements. Through comparing the metabolic energy consumption for Case $B$ and Case $E$ in Table 2, it turns out that Case $E$ consumes more energy than Case $B$, which indicates the arm orientation has certain influence on energy consumption.

As written above, the shorter trajectory required a bit more energy than longer one in Case $C$. There must be some other aspects interacting with trajectory to influence the metabolic energy consumption. Comparisons of all the cases' elbow and shoulder joint angle to energy property show us that the arm orientation is a big factor in influencing the energy consumption, especially in Case $C$. There is a big peak in the plot of shoulder joint angle of Case $A$ (the gray dash line in Fig. 5(e)), also big peaks in the plots of elbow joint angle of Case $B$, Case $D$, and Case $E$ (the gray solid line in Fig. 7(d), 9(a), and 10(a) respectively). However, there is no peak in the elbow and shoulder joint angles (Fig. 8(a),(b)). It requires more metabolic energy cost for the arm to pass through the joint angle peak. 

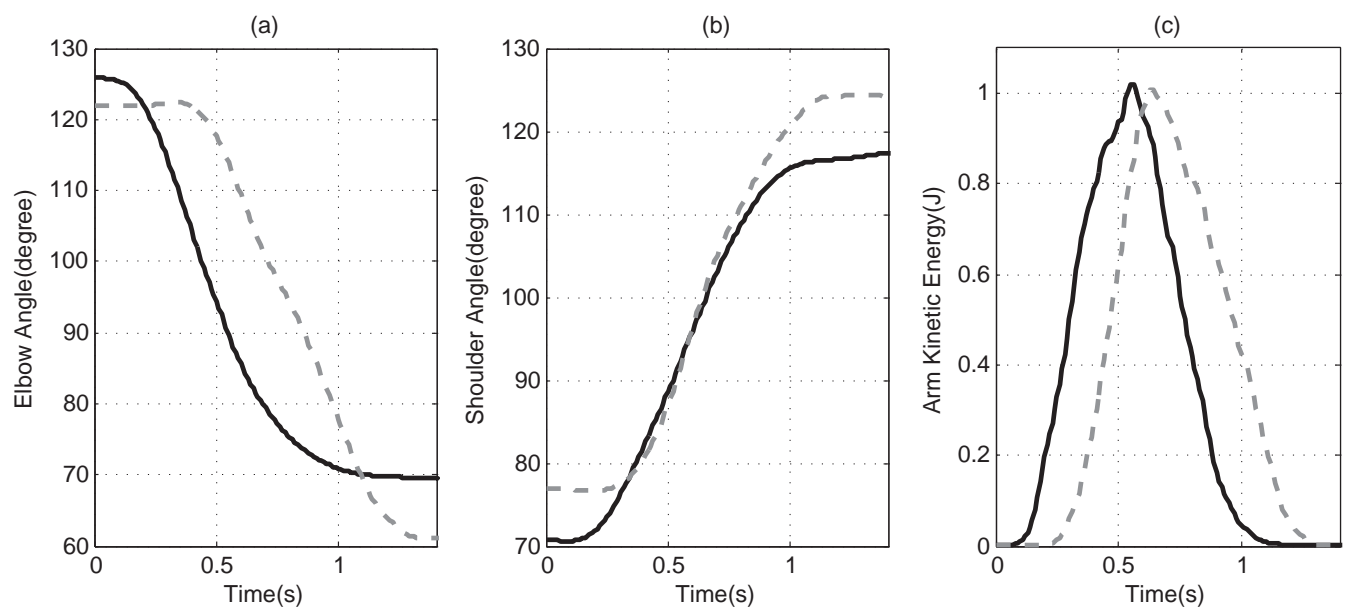

Figure 8: Measured elbow, shoulder joint angles and energy property profiles of two trajectories in Case $C$ : (a) elbow joint angle plots, (b) shoulder joint angle plots, (b) arm kinetic energy profiles.
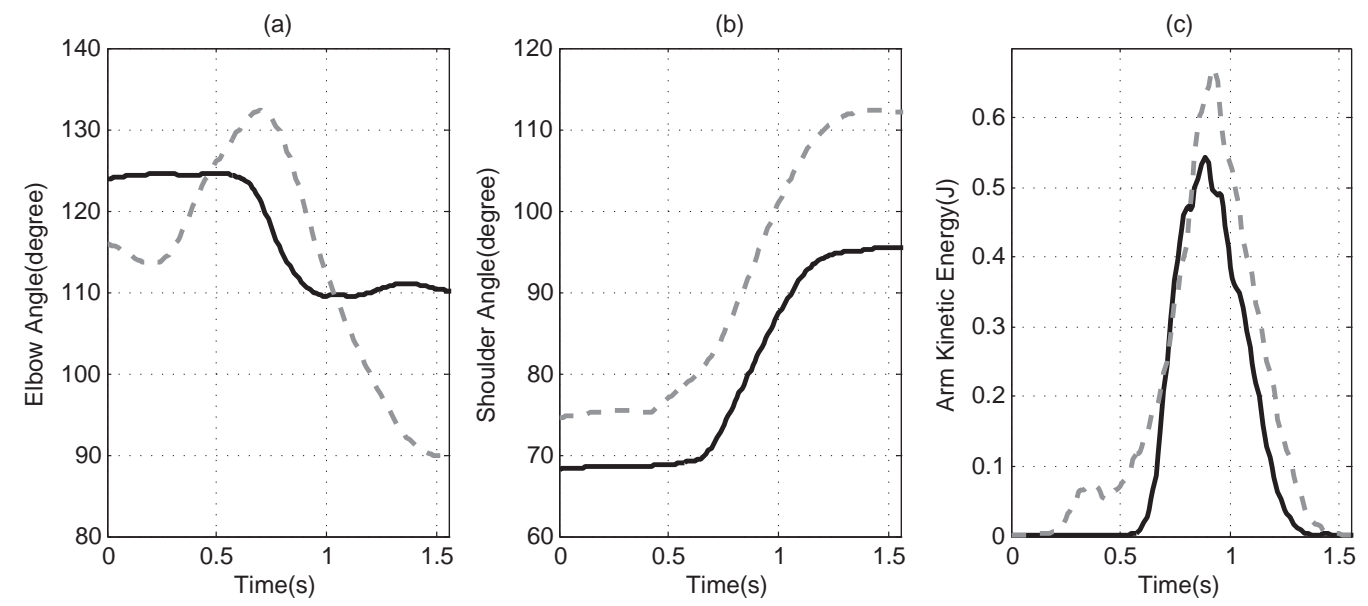

Figure 9: Measured elbow, shoulder joint angles and energy property profiles of two trajectories in Case $D$ : (a) elbow joint angle plots, (b) shoulder joint angle plots, (b) arm kinetic energy profiles.

\section{Discussion}

The experiments and results presented in this paper reveal the influences of the trajectory of arm motion, acceleration, and arm orientation on the metabolic energy consumption in human arm planar movements. The energy consumption has a great dependence on trajectory. However, this does not always mean the longer trajectory will consume more energy than the shorter one. Trajectory should be the first major factor in governing the energy consumption but not the only one. The ways that human brain conducting arm movements can be regarded as control strategies, such as minimum jerk theory, minimum travel cost theory, averaged specific power theory and so on. The fact that arm trajectories are seldom performed twice in exactly the same way can be used to argue that different strategies are used each time a movement is generated (Flash and Hogan, 1985). However, among these strategies, there should be one playing a major role in conducting movements, which might be the minimum metabolic energy cost theory (Alexander, 1997).

The experiments demonstrate the relationship between hand acceleration and metabolic energy cost. Hand acceleration is such a non-obvious factor that we should take advantage of data analyzing software to access the profile. The result emerges clearly from the data that acceleration has a positive correlation with the metabolic energy consumption.

It is noticed that arm orientation has certain impact on arm metabolic energy consumption. It requires more metabolic energy for the arm to pass through 

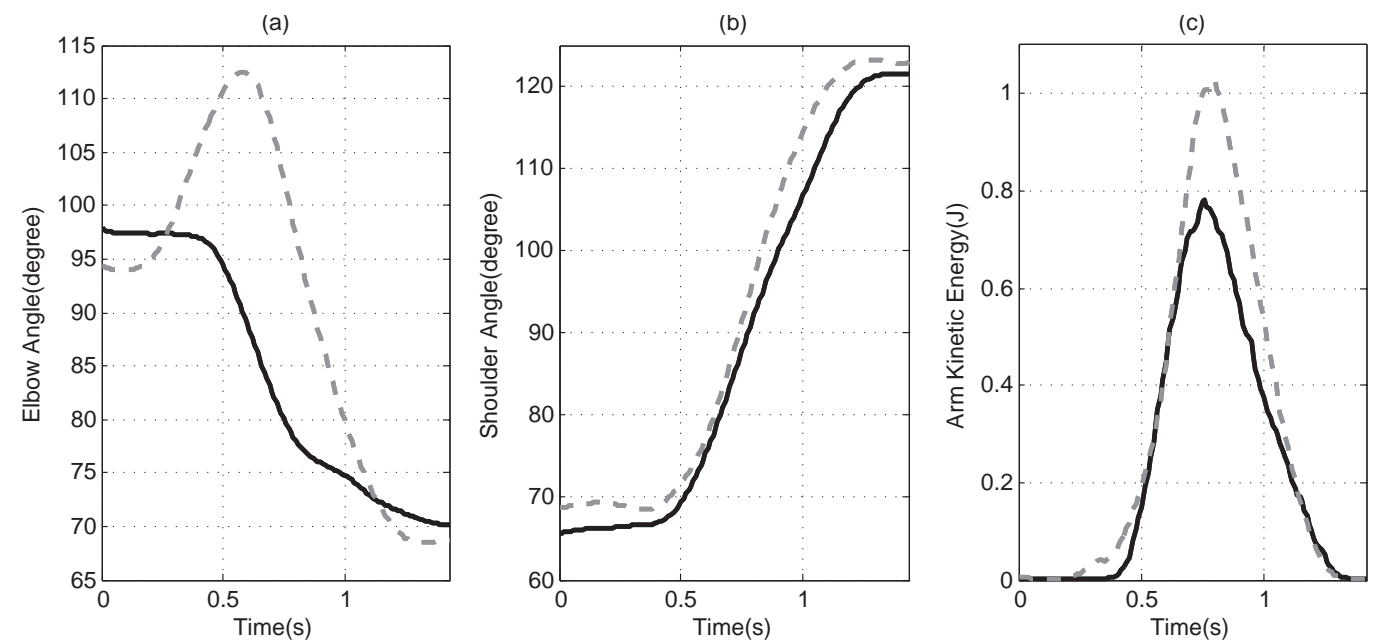

Figure 10: Measured elbow, shoulder joint angles and energy property profiles of two trajectories in Case $E$ : (a) elbow joint angle plots, (b) shoulder joint angle plots, (b) arm kinetic energy profiles.

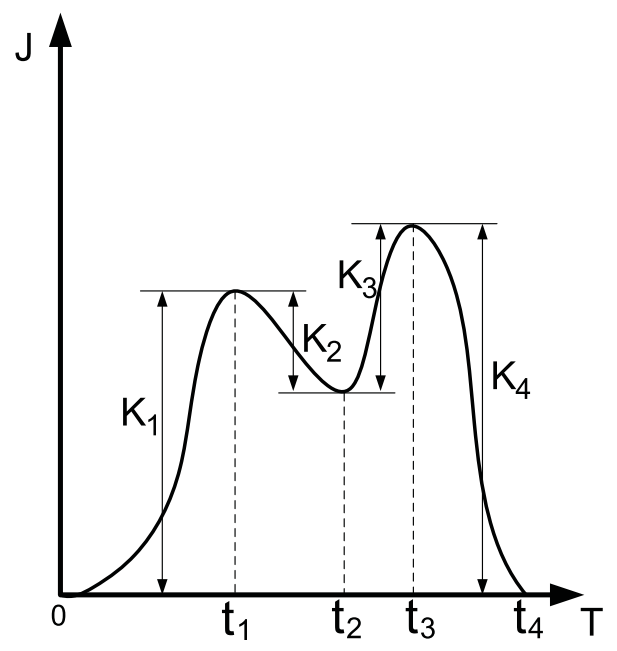

Figure 6: Method of calculating the muscle metabolism upon the arm kinetic energy profile.

the joint angle peak. Whether the elbow and shoulder joints have an angle peak, depends on the orientation that arm has chosen.

While the experiments were conducted in planar movement case only, solid conclusions are not made yet. To fully understand the characteristic of the human arm motions, more experiments should be conducted on more general and complex cases in ADL(Activities of Daily Living).
Table 2: Metabolic energy consumption for different paths

\begin{tabular}{ccc}
\hline Case & Path & Energy consumption \\
\hline \multirow{2}{*}{ A } & 1 & $0.73 J$ \\
& 2 & $3.74 J$ \\
& 3 & $1.39 J$ \\
\hline \multirow{2}{*}{ B } & 1 & $0.64 J$ \\
& 2 & $1.33 J$ \\
\hline \multirow{2}{*}{ C } & 1 & $2.33 J$ \\
& 2 & $2.03 J$ \\
\hline \multirow{2}{*}{ D } & 1 & $1.14 J$ \\
& 2 & $1.45 J$ \\
\hline \multirow{2}{*}{ E } & 1 & $1.78 J$ \\
& 2 & $2.26 J$ \\
\hline
\end{tabular}

\section{Conclusions}

In this paper, human arm motion in planar movements were studied by using Qualisys motion capture system. The motion data was analyzed and compared between each other. The kinematic and dynamic properties of human arm were obtained. The study indicates the relationship between the metabolic energy consumption and the trajectory of arm motion, and further, the acceleration and arm orientation contributions. Optimal trajectory with minimum metabolic energy consumption is identified. The optimal trajectory can find its application in robotic arm design where energy consumption is critical. More experiments are planned to 
study spatial arm motion and motion with specific load in hand.

\section{Acknowledgements}

The authors acknowledge the discussion with John Rasmussen, Aalborg University, Denmark. L. Zhou acknowledges the support by the National Natural Science Foundation of China (61603216). L. Zhou also acknowledges the support by the Fundamental Research Funds of Shandong University (2016TB008).

\section{References}

Abend, W., Bizzi, E., and Morasso, P. Human arm trajectory formation. Brain, 1982. 105:331-348. doi:10.1093/brain/105.2.331.

Alexander, R. M. A minimum energy cost hypothesis for human arm trajectories. Biological Cybernetics, 1997. 76(2):97-105. doi:10.1007/s004220050324.

Anglin, C. and Wyss, U. P. Review of arm motion analyses. Proc. Inst. Mech. Eng, 2000. 214(5):541555. doi:10.1243/0954411001535570.

Atkeson, C. G. and Hollerbach, J. M. Kinematic features of unrestrained vertical arm movements. Journal of Neuroscience, 1985. 5(9):2318-2330.

Buchanan, T. S. and Shreeve, D. A. An evaluation of optimization techniques for the prediction of muscle activation patterns during isometric tasks. Journal of Biomechanical Engineering, 1996. 118:565-574. doi:10.1115/1.2796044.

Fagg, A. H., Shah, A., and Barto, A. G. A computational model of muscle recruitment for wrist movements. Journal of Neurophysiology, 2002. 88:33483358. doi:10.1152/jn.00621.2002.

Flash, T. and Hogan, N. The coordination of arm movements: An experimentally confirmed mathematical model. Journal of Neuroscience, 1985. $5(7): 1688-1703$.

Georgopoulos, A. P., Schwartz, A. B., and Kettner, R. E. Neuronal population coding of movement direction. Science, 1986. 233:1416-1419. doi:10.1126/science.3749885.

Kashima, T. and Isurugi, Y. Trajectory formation based on physiological characteristics of skeletal muscles. Biological Cybernetics, 1998. 78:413-422. doi:10.1007/s004220050445.
Kashima, T., Isurugi, Y., and Shima, M. An optimal control model of a neuromuscular system in human arm movements and its contrl characteristics. Artificial Life Robotics, 2002. 6:205-209. doi:10.1007/BF02481269.

Kontaxis, A., Cutti, A., Johnson, G., and Veeger, H. A framework for the definition of standardized protocols for measuring upper-extremity kinematics. Clinical Biomechanics, 2009. 24:246-253. doi:10.1016/j.clinbiomech.2008.12.009.

Lan, N. Analysis of an optimal control model of multijoint arm movements. Biological Cybernetics, 1997. 76:107-117. doi:10.1007/s004220050325.

Ma, S. and Zahalak, G. I. A distribution-moment model of energetics in skeletal muscle. Journal of Biomechanics, 1991. 24(1):21-35. doi:10.1016/00219290(91)90323-F.

Morasso, P. Spatial control of arm movements. Experimental Brain Research, 1981. 42:223-227. doi:10.1007/BF00236911.

QTM. Qualisys track manager user manual. 2008.

Rosenbaum, D. A., Loukopoulos, L. D., Meulenbroek, R. G. J., Vaughan, J., and Engelbrecht, S. E. Planning reaches by evaluating stored postures. Psychological Review, 1995. 102(1):28-67. doi:10.1037/0033-295X.102.1.28.

Secco, E. L., Valandro, L., Caimmi, R., Magenes, G., and Salvato, B. Optimization of two-joint arm movements: a model technique or a result of natural selection? Biological Cybernetics, 2005. 93:288-306. doi:10.1007/s00422-005-0003-2.

Winter, D. A. Biomechanics and motor control of human movement. Wiley, 1990.

Winters, J. M. and Stark, L. Analysis of fundamental human movement patterns through the use of indepth antagonistic muscle models. IEEE Transactions on Biomedical Engineering, 1985. 32:826-839. doi:10.1109/TBME.1985.325498.

Woledge, R. C., Curtin, N. A., and Homsher, E. Energetic aspect of muscle contraction. Monographs of the Physiological Society, 1985. 41:1-357.

Zhou, L., Bai, S., Hansen, M. R., and Rasmussen, J. Modeling of human arm energy expenditure for predicting energy optimal trajectories. Modeling, Identification and Control, 2011. 31(3):91-101. doi:10.4173/mic.2011.3.1. 